\title{
Architecture and Adoption Model for Cloud in Higher Education: Indian Perspective
}

\author{
Vaishali Pardeshi
}

\begin{abstract}
Cloud implementation is seen as a possible rescue measure to the financial crisis faced by higher education (HE) institutes due to the shrinking IT budgets and escalating IT needs. In this paper, architecture for implementing cloud in the HE institute involving various Deployment Models and Service Models is proposed. A five step framework based on Roger's Innovation-Diffusion model has been suggested for adopting cloud in HE institutes. For smoothing the migration from traditional system to cloud based system a five phase strategy is presented. Finally the challenges and benefits reaped by $\mathrm{HE}$ institute form cloud adoption are identified.
\end{abstract} SaaS

Keywords--- Cloud, Rogers Diffusion Model, IaaS, PaaS,

\section{INTRODUCTION}

$\mathrm{H}$ IGHER education (HE) plays a crucial role in overall development of a country, as it is considered as a powerful means of building a knowledge based society. The collaboration between universities, government organizations, industries, researchers and students has proven their contribution for transformation of the society and the entire world economy [1]. In India HE is facing challenges in terms of access, equity and quality.

India has the largest number of HE institutes in the world and is third largest in terms of student enrolment (after China and USA). As per UGC and MHRD Reports 2012, India has 42 central universities, 160 private universities, 275 state universities and approximately 31,000 autonomous colleges offering HE [2]. There are 14.6 million students undergoing $\mathrm{HE}$ in India as of 2011 and is expected to be 40 million by 2020. The infrastructure would be insufficient to meet the growing demand. There would be a requirement of 25 million additional seats in the next decade. Additionally, faculty shortage, ill-equipped libraries and outdated curricula impair the HE system [3].

During the last few years, the universities offering $\mathrm{HE}$ are making transition to research universities [4,5] and these universities use IT infrastructure as a foundation for their educational and research activities. With the evolution of technology, number of educational services migrates from traditional form to the online form. These educational services, requires an adequate IT infrastructure using the appropriate technologies, guaranteeing access to a large number of users, using fast and secure service access. The

Vaishali Pardeshi, Assistant Professor, Institute of Management, MET, Bandra, Mumbai, India.E-mail:vaishaliincthane@gmail.com adaptation of new technology is very slow mainly due to the cost implications. These transitions requires massive funding and investment, which are difficult to come at the times of deep recession and depleted budget reserves of government and private institutions. The funding offered to HE institutes has sharply decreased in times of economic slowdown, leading to financial crisis in HE institutions. To address the financial shortfall, HE institutes are resorting to a variety of cost-cutting measures, including significant cuts to IT budgets.

The HE institutes also requires huge investments in the form of ever increasing costs of books, journals and educational software that form the backbone for imparting educational services in any economy. The enormous amount of investments is difficult not only to the emerging economies, but also to the developed economies which struggle to provide it [6]. In the past few years the concept of "Cloud Computing (Cloud)" has emerged as a viable and promising solution to the challenges associated with shrinking IT budgets and escalating IT needs. Cloud is a model for enabling convenient, on-demand network access to a shared pool of configurable computing resources (e.g., networks, servers, storage, applications, and services) that can be rapidly provisioned and released with minimal management effort or service provider interaction. Users can access these resources from any computer with a high speed Internet connection, while having no other connection to the hardware that holds the source software [7]. Because computation takes place on a remote server, the user's hardware and software requirements are much lower than they would be otherwise, reducing both cost and maintenance requirements [8]. For this reason, Cloud holds appeal for HE institutions seeking to reduce IT budgets. The usage of cloud computing can reduce drastically the investments required [9].

\section{Cloud COMPUTING MODELS}

Service models describe what kind of services can be obtained from the cloud. Cloud service delivery is divided among three typical models and various derivative combinations. The three fundamental classification of the cloud service model is shown in figure 1. This is often referred to as the "SPI Model," where 'SPI' refers to Software, Platform or Infrastructure (as a Service), respectively. Depending on the model selected, the cloud provider delivers differentiated services. These services are generally classified according to the level of the IT architecture they reside on. The cloud provider determines how the service is offered within the agreed upon service level agreements (SLA), and how the services on underlying layers may be accessed. Each service model consists of the following layers [10]: 
- Applications: The applications used by a customer based on the agreement

- Runtime: The environment in which the particular application is running, including the runtime library of the application's requisite functions.

- Middleware: The switching software and/or middleware serve as a communication with other applications, databases and the operating system.

- Operating System: The operating system provides and manages the system resources of the underneath hardware to the user.

- Hardware: The hardware consists of the physical units, such as servers, CPU, storage, and the network. It may contain a virtualization layer that provides the virtualized infrastructure resources to the OS.
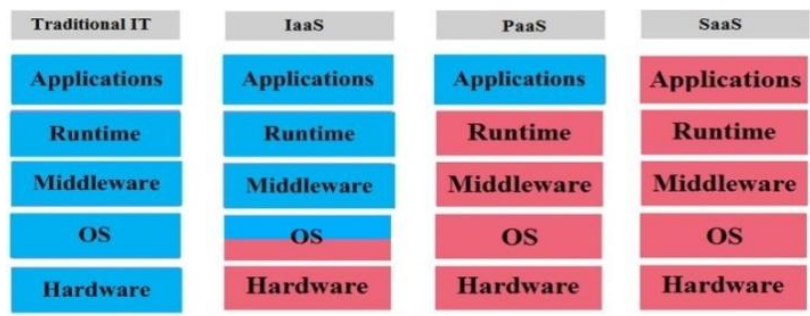

Figure 1: Service Models for Cloud. Blue Indicates as level Owned and Operated by the organization, and red indicates levels run and operated by the service provider

\section{A. Cloud Service Models}

- Infrastructure as a Service (IaaS): can be used to satisfy the infrastructure needs of the students, faculties or researcher, globally or locally requiring some specific hardware configuration for a specific task. It provides a remote virtual hosting server for file storage, as it enables a user to save all of their file types in a virtual host and retrieve them from anywhere with an internet connection. Examples of IaaS are Google Compute Engine, Google Cloud Storage and Google Big Query.

- Platform as a Service (PaaS): certain providers are opening up application platforms to permit customers to build their own application without the cost and complexity of buying and managing the underlying hardware and software layers. PaaS provides the entire infrastructure needed to run applications over the Internet, PaaS is based on a metering or subscription model so users only pay for what they use. Examples of PaaS are Google App Engine, Force.com and Heroku.

- Software as a Service (SaaS): the application service provider is hosting the application which runs and interacts through web browser, hosted desktop or remote client. It eliminates the need to install and run the application on customers own computer, thus simplifying maintenance and support services. Organizations that operate on SaaS are not burdened with the time-consuming and costly task of managing software updates, security patches and a host of other administrative duties for on-premise software solutions. SaaS ensures that these tasks are managed quickly, efficiently and affordably on the back-end.

\section{B. Cloud Deployment Models}

Regardless of the service model utilized (SaaS, PaaS, or IaaS) there are four deployment models for cloud services, with derivative variations that address specific requirements:

- Private cloud: The cloud infrastructure is provisioned for exclusive use by a single organization comprising multiple consumers. It may be owned, managed, and operated by the organization, a third party, or some combination of them, and it may exist on or off premises.

- Community cloud: The cloud infrastructure is provisioned for exclusive use by a specific community of consumers from organizations that have shared concerns (e.g., mission, security requirements, policy, and compliance considerations). It may be owned, managed, and operated by one or more of the organizations in the community, a third party, or some combination of them, and it may exist on or off premises.

- Public cloud: The cloud infrastructure is provisioned for open use by the general public. It may be owned, managed, and operated by a business, academic, or government organization, or some combination of them. It exists on the premises of the cloud provider.

- Hybrid cloud: In this case the cloud infrastructure is a composition of two or more distinct cloud infrastructures (private, community, or public) that remain unique entities, but are bound together by standardized or proprietary technology that enables data and application portability (e.g., cloud bursting for load balancing between clouds)"[11].

In addition, it is possible to have derivative cloud deployment models emerging due to the maturation of market offerings and customer demand. For example, virtual private

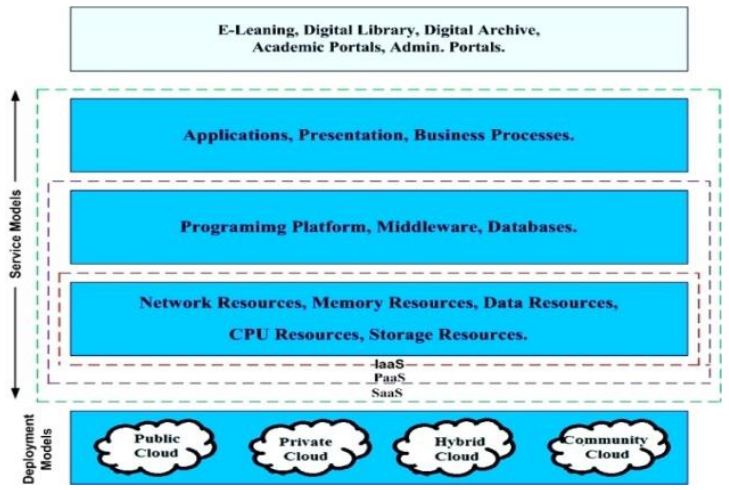

Figure 2: Architecture for Cloud Implementation in HE Institutes

Clouds - utilizes public cloud infrastructure in a private or semi-private manner. In virtual private cloud the public cloud resources are interconnected to the internal resources of a consumers' datacenter, usually via virtual private network (VPN) connectivity [12]. The cloud system architecture design has clear implications on the future flexibility, security, and mobility of the resultant solution, as well as its collaborative 
capabilities. As a rule of thumb, perimeterized solutions are less effective than de-perimeterized solutions in each of the four areas [12]. Outmost consideration should also be given while choosing between proprietary and open solutions.

\section{Proposed Architecture for HE Institutes}

In this section we have proposed a cloud based IT architecture for a $\mathrm{HE}$ institute (figure 2). The IaaS is the foundation of all cloud services, with PaaS build upon IaaS, and followed by SaaS build upon PaaS as shown in the architectural diagram. A clear understanding of the relationships and dependencies between various cloud models is important for understanding security risks. The capabilities and security risk are inherited from layer to layer. IaaS contains the infrastructure resource and the hardware platforms. IaaS provides the physical and logical connectivity between the hardware resources. IaaS contains a set of APIs which allow management and other forms of interaction with the infrastructure by consumers [12]. PaaS provides additional layer containing programming environment, middleware capabilities, database, messaging, and queuing. This allows cloud users/developers to build their applications on the cloud platform. The SaaS is build upon the underlying IaaS and PaaS stacks. SaaS provides the application level capabilities to users. It includes functions for presentation, application, and provides management capabilities. It should noted that there are significant trade-offs to each model in terms of integrated features, complexity vs. openness (extensibility), and security [12].

\section{Current Scenario of Cloud Computing in $\mathrm{HE}$}

According to CDW'S 2013 state of the cloud report, the percentage of $\mathrm{HE}$ institutes implementing or maintaining the cloud was $37 \%$ in 2011 and has grown to $44 \%$ in 2012 [13]. According to Gartner and IDC by 2015, smartphones and tablets will be two-third of the overall existing devices. This increase will demand massive storage requirement in cloud. By 2020 Seagate projects, $61 \%$ of the whole data will be shifted to cloud, of which two-third will be on personal cloud [14]. The percentage of services and applications moving to cloud in $\mathrm{HE}$ sector are $31 \%, 29 \%$ and $25 \%$ for storage, messaging/conferencing and collaboration, and computing power respectively [13]. Acknowledging the need and potential leading IT providers are coming up with cloud based software for HE institutes. The cloud-computing market is projected to grow from $\$ 40.7 \mathrm{~B}$ in 2011 to $\$ 240 \mathrm{~B}$ in 2020 [15]. The trend in HE sector is clearly shifting towards cloud services adoption. According to a 2011 study by CDW, only $5 \%$ percent of U.S. college and university respondents were not considering a cloud migration. About $29 \%$ had developed a written strategic plan for the adoption of cloud computing, with 28 percent in the midst of implementation [16]. The HE Funding Council for England has developed a new program that would invest up to $£ 10$ million in cloud computing and shared IT infrastructure for universities and colleges [17].

\section{ROGERS DIFFUSION MODEL}

One of the most popular adoption models is the Roger's Innovation-Diffusion model and this model is used by a broad variety of disciplines. Dooley [18] and Stuart [19] have mentioned several of these disciplines such as political science, public health, communications, history, economics, technology, and education. Everett M. Rogers in the book Diffusion of Innovations [20] mentions the innovationdiffusion in five stages as mentioned below. Innovation diffusion framework can be used for adopting cloud in the Indian HE space.

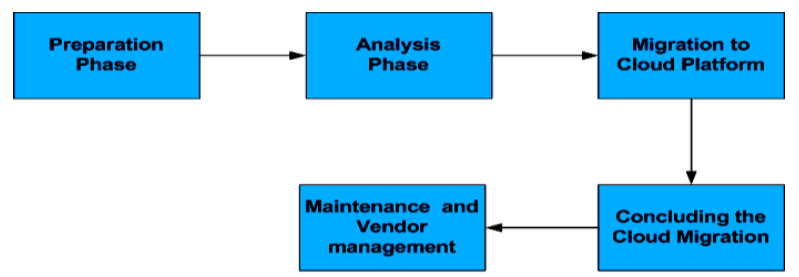

Figure 3: Five Stage Models for Cloud Adoption based on Rogers Innovation Diffusion Model

- Knowledge Stage: According to Rogers Model the innovation-decision process starts with the knowledge stage. In this step, an individual learns about the existence of innovation and seeks information about the innovation. "What?," "how?," and "why?" are the critical questions in the knowledge phase. During this phase, the individual attempts to determine "what the innovation is and how it is and why it works". According to Roger, the questions form three types of knowledge: (1) awareness-knowledge, (2) how-to-knowledge, and (3) principles-knowledge.

Awareness knowledge represents the knowledge of the innovation's existence. This type of knowledge can motivate the individual to learn more about the innovation and, eventually, to adopt it. The government of India and the department of $\mathrm{HE}$ should make the educational institutions aware of the existence of cloud computing, its opportunities and challenges, its functions and principles.

How-to-knowledge contains information about how to use an innovation correctly. As Wetzel [21] stated, even the faculty who have technical backgrounds may not use technology in teaching, if they do not have knowledge of how to use it correctly. Roger saw this knowledge as an essential variable in the innovation-decision process. To increase the adoption chance of an innovation, an individual should have a sufficient level of how-toknowledge prior to the trial of this innovation. Thus the next step is to train the faculties and the staff on using the technology.

Principles-knowledge includes the functioning principles, describing how and why an innovation works. An innovation can be adopted without this knowledge, but the misuse of the innovation may cause its discontinuance. To create new knowledge, technology education and practice should provide not only a how-to experience but also a know-why experience [22]. An individual may have all the necessary knowledge, but this does not mean that the individual will adopt the innovation. Thus, the next step is to make faculty and staff aware of the principles and functioning of the cloud.

- Persuasion Stage: The persuasion step occurs when the individual has a negative or positive attitude toward the innovation. The individual shapes his or her attitude after he or she knows about the 
innovation. The persuasion stage follows the knowledge stage in the innovation-decision process. A top-down approach can be followed. To start with, the Directors/Top level Management of premier institutes needs to be convinced about the benefits of cloud adoption. Once they have adopted the others will follow the suit.

- Decision Stage: In the decision stage, the individual chooses to adopt or reject the innovation. If an innovation has a partial trial basis, it is usually adopted more quickly, since most individuals first want to try the innovation in their own situation and then come to an adoption decision. Educational institutes can shift some of their infrastructure to the cloud on a pilot basis. Monitoring of the savings and benefits, accrued will make decision making easier.

- Implementation stage: At the implementation stage, an innovation is put into practice. Some of the daily necessities of the educational institutes are shifted to the cloud. To start with, email servers can be moved to the cloud. Once the pilot project involving the premier institutes is successful, the scale of implementation may be increased. After using the technology new ideas may come up leading to reinvention. Reinvention is "the degree to which an innovation is changed or modified by a user in the process of its adoption and implementation" [20]. The more reinvention takes place, the more rapidly an innovation is adopted and becomes institutionalized.

- Confirmation Stage: In India, there is a hierarchy in $\mathrm{HE}$ with central and state universities having affiliated colleges under them. The decisions are taken by the government and administration. The universities can decide to introduce cloud computing in their affiliated colleges. Incentives for early adoption may be given by government.

\section{Challenges AND BenEFITS OF ClOUd IMPLEMENTATION TO THE HE INSTITUTES}

\section{A. Challenges for Cloud Adoption}

- Establishing trust is a major challenge for cloud services in HE sector. Trust involves the expectation about availability, privacy and IPR.

- The concerns about security breaches are the biggest obstacles to cloud adoption in HE sector. The possible security risks include the loss of governance, isolation failure, compliance risks, data protection, incomplete or insecure data deletion and malicious insider.

- The concerns related to the privacy, data integrity, intellectual property management and regulation issues.

- The risk assessment of cloud adoption in HE is a critical task. Some HE institutes feel that many of the risks related to cloud is transferred to the cloud vendor/service provider. Some third party may be involved for such assessment.

- Flexibility of the HE institute's IT infrastructure in adopting cloud is a major challenge.
- The HE institutions need to standardize their processes to exploit the cost advantage. This is something that the $\mathrm{HE}$ institutes have found difficult to do.

- Customizability: HE institutes typically use their own in-house applications with a portion that is customized to their own IT lab structure. The major concern is the transitioning of applications to the cloud environment and how much of the customizability will be lost in that process.

\section{B. Benefits of Cloud adaptation}

- Fiscal advantages: According to the 2011 CDW survey report, U.S.-based HE institutes have saved about $21 \%$ by migrating applications to the cloud. This is attractive when we consider the underutilized infrastructure during vacation breaks. By using a virtualization model and shared infrastructure, cloud adoption promises reduced expenses.

- Increased efficiency and availability: The cloud model provides the ability to rapidly acquire, provision, and deploy new IT platforms, services, applications, and test environments. Months-long procurement process are reduced to days, University networks are securely available, regardless of the circumstances. The organization becomes more agile and efficient, and can quickly respond to changing environmental requirements.

- Simplification and standardization: The cloud consolidates and simplifies the datacenters and facilities. It standardizes practices and improves compliance with regard to security. Cloud implementations enable universities to build a solution at once and then use that solution multiple times. This lowers costs, increases reliability, and reduces time for implementation.

- Innovation: The primary purpose of technology should be to enable and inspire innovation in the classroom and lab. That means giving educators, administrators, and students the freedom they need to do their work. With the agility of the cloud, HE institutes can try out new applications with minimal commitment, pay for as much as they use, and adjust as necessary. The cloud helps institutions to explore up-to-the-minute research opportunities by sharing cloud-computing resources with other universities.

\section{CONCLUSION}

Cloud is an emerging technology paradigm that promises to provide solution to the current financial crisis faced by $\mathrm{HE}$ institutes. The movement from traditional system towards cloud would enable the HE institutions to cope with rapidly changing software and hardware needs at lower cost. We have proposed a cloud based IT architecture for a HE consisting of the IaaS as a foundation layer, with PaaS build upon IaaS, and followed by SaaS build upon PaaS. A clear understanding of the relationships and dependencies between various cloud models is important for understanding security risks. The Roger's model used for cloud adoption in HE institute is a 
popular model for innovation and diffusion of any new technology. The model consists of five step knowledge, persuasion, decision, implementation and confirmation. Finally the challenges and benefits reaped by HE institute form cloud adoption are stated

\section{ACKNOWLEDGMENT}

The author would like to thank Mumbai Education Trust's (MET), Institute of Management, Mumbai for providing opportunity to carry out this research and for providing financial support and assistance.

\section{REFERENCES}

[1] Lazowska E, Lee P, Elliott C and Smarr L, Infrastructure for Escience and Elearning in Higher Education, Computing Community Consortium, http://www.cra.org/cloudc/docs/init/ Infrastructure.pdf, 2008.

[2] Higher Education in India: Twelfth Five Year Plan (2012-2017) and beyond, FICCI Higher Education Summit 2012, published by Ernst \& Young Pvt. Ltd, 2012.

[3] IT/ICT Adoption in Indian Higher Education, white paper Calsoft Labs, 2012.

[4] Mircea M, GhilicMicu B, and Stoica M, Combining Knowledge, Process and Business Intelligence to Delivering Agility in Collaborative Environment, Spotlight on Business Intelligence, Future Strategies Inc. \& Workflow Management Coalition, Florida, 2010.

[5] Bozzelli T, Will the Public Sector Cloud Deliver Value? Powering the Cloud Infrastructure, CISCO, http://www.cisco.com/web/strategy/ docs/ gov/2009_cloud_public_sector_tbozelli.pdf, 2009.

[6] St John E P, and Parsons M D, Public funding of higher education, Baltimore and London: The John Hopkins University Press, 2004.

[7] Gruman G, What cloud computing really means. InfoWorld, http://www.info-world.com/print/34031, 2008.

[8] Erenben C, Cloud computing: the economic imperative. ESchool News, vol. 12, no. 3, pp. 13-19, 2009.

[9] Nabil Sultan, Cloud computing for education: A new dawn?, International Journal of Information Management, vol 30, , pp. 109116, 2010.

[10] Matthias Kaiserswerth, Olivier Brian, Thomas Brunschwiler, et. al., Cloud Computing, SATW White Paper Cloud Computing, pp. 1-55, 2012.

[11] Peter Mell and Timothy Grance, The NIST Definition of Cloud Computing, National Institute of Standards and Technology, US department of commerce, Special Publication 800-145, pp.1-3, 2011.

[12] Glenn Brunette, Rich Mogull, et. al., Security Guidance for Critical Areas of Focus in Cloud Computing V2.1, Cloud Security Alliance, pp 1-70, 2009.

[13] Kelly Caraher and Martin Nott, Silver linings and surprises, CDW's 2013 State of The Cloud Report, pp. 1-32, 2013

[14] Jan-Martin Lowendahl, A Quick Look at Cloud Computing in Higher Education, Gartner, 2012

[15] Stefan Ried and Holger Kisker, Sizing the Cloud, Forrester Research, 2011.

[16] From Tactic to Strategy: The CDW 2011 Cloud Computing Tracking Poll, 2011.

[17] http://www.hefce.ac.uk/news/hefce/2011/cloud.htm.

[18] Dooley K E, Towards a holistic model for the diffusion of educational technologies: An integrative review of educational innovation studies", Educational Technology \& Society, vol. 2, no 4, pp. 35-45, 1999.

[19] Stuart W D, Influence of sources of communication, user characteristics and innovation characteristics on adoption of a communication technology (Doctoral dissertation, The University of Kansas, 2000). ProQuest DigitalDissertations. (UMI No. AAT 9998115), 2000.

[20] Everett M. Rogers, Diffusion of Innovations, Free Press, 5th Edition, New York, 2003.

[21] Ismail Sahin, Detailed review of Rogers' diffusion of innovations theory and educational technology-related studies based on Rogers' theory, The Turkish Online Journal of Educational Technology - TOJET, vol. 5, No 2, pp 14-23, 2006.

[22] Seemann K, Basic principles in holistic technology education, Journal of Technology Education, vol. 14, no. 2, pp. 28-39, 2003.

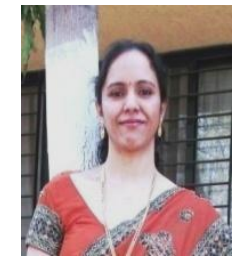

Vaishali Pardeshi received M. Phil, M.C.M from YCMOU and Dr BAM University in 2009 and 2003 respectively. She has also done M.B.A for Sikkim manipal university. She has published/presented eight papers in reputed Journal/ conferences. She is presently working as Asst. Professor in the Institute of Management, Mumbai Education Trust (MET), Mumbai, India. She has 10 years of rich teaching \& research experience in IT and management studies. Her research area includes cloud computing implementation for higher education sector, Big Data, mobile computing, social BI, etc. 